Discrete Comput Geom 38:305-319 (2007)

DOI: $10.1007 / \mathrm{s} 00454-007-1340-9$

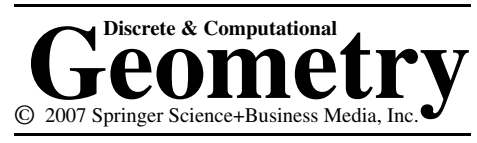

\title{
Typical Cells in Poisson Hyperplane Tessellations*
}

\author{
Daniel Hug ${ }^{1}$ and Rolf Schneider ${ }^{2}$ \\ ${ }^{1}$ Fachbereich Mathematik, Universität Duisburg-Essen, \\ Campus Essen, D-45117 Essen, Germany \\ daniel.hug@uni-due.de \\ ${ }^{2}$ Mathematisches Institut, Albert-Ludwigs-Universität, \\ D-79104 Freiburg i. Br., Germany \\ rolf.schneider@math.uni-freiburg.de
}

\begin{abstract}
It is proved that the shape of the typical cell of a stationary and isotropic Poisson random hyperplane tessellation is, with high probability, close to the shape of a ball if the $k$ th intrinsic volume $(k \geq 2)$ of the typical cell is large. The shape of typical cells of large diameter is close to the shape of a segment.
\end{abstract}

\section{Introduction}

The zero cell of a stationary Poisson hyperplane tessellation is a frequently studied type of random polytope. It is generated in the following way. Let $X$ be a stationary and isotropic Poisson process in the space of hyperplanes of $\mathbb{R}^{d}$. It induces, in the obvious way, a random tessellation of $\mathbb{R}^{d}$ and thus a process $Y$ of $d$-dimensional polytopes tiling $\mathbb{R}^{d}$, called the cells of the tessellation. The almost surely unique cell $Z_{0}$ containing the origin $\boldsymbol{o}$ is called the zero cell. (Replacing the origin $\boldsymbol{o}$ by a different fixed point $\boldsymbol{t}$ would result in a random polytope stochastically equivalent to $Z_{0}+t$, by the stationarity of $X$.)

Another type of random polytope associated with a stationary Poisson hyperplane tessellation is the typical cell $Z$. The idea behind this is roughly as follows. One considers a large compact region of the tessellation and picks out a cell at random, where each cell within that region has the same chance of being picked, and then translates the chosen cell appropriately; this yields a realization of the typical cell (or, rather, of its translation class). A precise definition is recalled in Section 3. The distinction between the two types of random polytopes was made clear, and both have been studied, in the work

\footnotetext{
* This work was supported in part by the European Network PHD, FP6 Marie Curie Actions, RTN, Contract
} MCRN-511953. 
of Miles [16], [17] and Matheron [13]. Matheron used to distinguish between the two random polytopes by calling their distributions respectively the "volume law" and the "number law" of a Poisson polyhedron. Other names found in the literature are "Crofton polytope" and "Poisson polytope", respectively.

It was conjectured by D. G. Kendall for the case of the plane that zero cells of large area must have a shape that is close to circular, with high probability. More precisely, his conjecture (see Foreword to the first edition of [25]) claimed that the conditional law for the shape of $Z_{0}$, given the volume $V_{2}\left(Z_{0}\right)$ of $Z_{0}$, converges weakly, as $V_{2}\left(Z_{0}\right) \rightarrow \infty$, to the degenerate law concentrated at the circular shape. A proof was given by Kovalenko [9], [11]. An extension to higher dimensions and to nonisotropic Poisson hyperplane processes (where the limit shapes are nonspherical) was obtained in [4]. Already Miles [19] had discussed, in the planar case, versions of Kendall's problem where the size is not measured by the volume, but by other functionals. In [5] zero cells with a large $k$ th intrinsic volume, $k \in\{2, \ldots, d\}$, or of large inradius were studied. Similar investigations concern typical cells of Voronoi mosaics induced by stationary Poisson point processes, see [10] for the planar case, [5] for higher dimensions, and [6] and [7] for typical cells of the dual Poisson-Delaunay tessellations. A very general version of Kendall's problem, comprising Poisson hyperplane processes which are not necessarily stationary, and admitting a quite general class of size functionals, is the subject of [8].

The results on stationary Poisson hyperplane tessellations and general size functionals so far all concerned the zero cell, with the exception of the volume case. In [4] the results on zero cells of large volume were transferred to typical cells of large volume, using the known fact that the distribution of the zero cell is, if translations are neglected, the volume-weighted distribution of the typical cell. For size functionals other than the volume, there seems to be no similarly direct transference principle.

In this paper we obtain results on the asymptotic shapes of large typical cells of stationary, isotropic Poisson hyperplane tessellations in $\mathbb{R}^{d}$, where "large" either means large $k$ th intrinsic volume, $k \in\{2, \ldots, d\}$, or large diameter. The asymptotic shapes are balls in the first case and segments in the second. As in the former papers, we show that large deviations of the shapes of large cells from the limit shapes have very small probability. The precise formulation requires a few preparations; the main result is the theorem in the next section.

\section{Preliminaries and Main Results}

The real Euclidean vector space $\mathbb{R}^{d}(d \geq 2)$ is equipped with the scalar product $\langle\cdot, \cdot\rangle$ and the induced norm $\|\cdot\|$. The space $\mathcal{K}^{d}$ of convex bodies (nonempty, compact, convex sets) is endowed with the Hausdorff metric. $B^{d}:=\left\{\boldsymbol{x} \in \mathbb{R}^{d}:\|\boldsymbol{x}\| \leq 1\right\}$ is the unit ball and $S^{d-1}:=\left\{\boldsymbol{x} \in \mathbb{R}^{d}:\|\boldsymbol{x}\|=1\right\}$ is the unit sphere of $\mathbb{R}^{d}$. We write $\kappa_{d}$ for the volume and $\omega_{d}=d \kappa_{d}$ for the surface area of the unit ball. The normalized spherical Lebesgue measure on $S^{d-1}$ is denoted by $\sigma$. We denote the space (with the usual topology) of hyperplanes in $\mathbb{R}^{d}$ by $\mathcal{H}^{d}$. We write

$$
H(\boldsymbol{u}, t):=\left\{\boldsymbol{x} \in \mathbb{R}^{d}:\langle\boldsymbol{x}, \boldsymbol{u}\rangle=t\right\}, \quad H^{-}(\boldsymbol{u}, t):=\left\{\boldsymbol{x} \in \mathbb{R}^{d}:\langle\boldsymbol{x}, \boldsymbol{u}\rangle \leq t\right\}
$$

for $\boldsymbol{u} \in S^{d-1}$ and $t \in \mathbb{R}$. Every hyperplane $H \in \mathcal{H}^{d}$ has a representation $H=H(\boldsymbol{u}, t)$; it is unique if $t>0$ and is then called the standard representation (the hyperplanes 
containing $\boldsymbol{o}$ can be neglected later). For a hyperplane $H$ and a point $\boldsymbol{x} \in \mathbb{R}^{d} \backslash H$, we denote by $H^{-}(\boldsymbol{x})$ the closed halfspace bounded by $H$ that contains $\boldsymbol{x}$; we write $H^{-}$for $H^{-}(\boldsymbol{o})$.

The underlying probability space is $(\Omega, \mathbf{A}, \mathbb{P})$, and the mathematical expectation is denoted by $\mathbb{E}$. Throughout this paper, $X$ is a stationary and isotropic Poisson hyperplane process in $\mathbb{R}^{d}$. Thus, $X$ is a Poisson point process in the space $\mathcal{H}^{d}$, and its distribution is invariant under translations and rotations (see [24] for an introduction). As usual, $X$ denotes a (random) simple counting measure as well as its support; thus, $X(\mathcal{A})$ and $\operatorname{card}(X \cap \mathcal{A})$ have the same meaning. The intensity measure $\Theta=\mathbb{E} X$ is of the form $\Theta=\lambda \mu$, where $\lambda>0$ is the intensity of $X$ and $\mu$ is the motion invariant measure on $\mathcal{H}^{d}$ given by

$$
\mu=2 \int_{S^{d-1}} \int_{0}^{\infty} \mathbf{1}\{H(\boldsymbol{u}, t) \in \cdot\} \mathrm{d} t \sigma(\mathrm{d} \boldsymbol{u}) .
$$

For $K \in \mathcal{K}^{d}$, let $\mathcal{H}_{K}:=\left\{H \in \mathcal{H}^{d}: H \cap K \neq \emptyset\right\}$, then $\mathbb{E} X\left(\mathcal{H}_{K}\right)$, the expected number of hyperplanes in the process hitting $K$, is given by $\mathbb{E} X\left(\mathcal{H}_{K}\right)=\lambda \Phi(K)$ with

$$
\Phi(K):=2 \int_{S^{d-1}} h(K, \boldsymbol{u}) \sigma(\mathrm{d} \boldsymbol{u}),
$$

where $h(K, \cdot)$ is the support function of $K$. The function $\Phi$ has been called the parameter functional in [8], since $\Phi(K) \lambda$ is the parameter of the Poisson distribution

$$
\mathbb{P}\left(X\left(\mathcal{H}_{K}\right)=n\right)=\frac{[\Phi(K) \lambda]^{n}}{n !} \mathrm{e}^{-\Phi(K) \lambda}, \quad n \in \mathbb{N}_{0} .
$$

In the isotropic case considered here, $\Phi$ is nothing but the mean width, but for easier comparison we keep the notation of [8].

We use $\Sigma$ to denote either the $k$ th intrinsic volume $V_{k}$ (so that $V_{d}$ is the volume and $2 V_{d-1}$ is the surface area), for $k \in\{2, \ldots, d\}$, or the diameter $D$. Then $\Sigma$ is a real function on $\mathcal{K}^{d}$ with the following properties: it is continuous, translation invariant, homogeneous of some degree $k>0$, and increasing under set inclusion. Moreover, there exists a constant $c_{1}>0$ such that $V_{d}(K) \leq c_{1} \Sigma(K)^{d / k}$. (For the intrinsic volumes, this follows from the Aleksandrov-Fenchel inequalities (see [23]), and for the diameter from the isodiametric inequality.) The subsequent investigations hold for any "size functional" $\Sigma$ with the listed properties.

It is clear (or see [8]) that $\Phi$ and $\Sigma$ satisfy an inequality

$$
\Phi(K) \geq \tau \Sigma(K)^{1 / k} \quad \text { for } \quad K \in \mathcal{K}^{d},
$$

with a constant $\tau>0$, where equality is attained by some bodies. Every convex body $K \in \mathcal{K}^{d}$ with more than one point for which equality holds is called an extremal body (for the given functional $\Sigma$ ). For $\Sigma=V_{k}(k \geq 2)$, the extremal bodies are the balls, and for $\Sigma=D$, they are the segments. This is well known, together with the explicit values of $\tau$.

As in [8], a real function $\vartheta$ on $\left\{K \in \mathcal{K}^{d}: \Sigma(K)>0\right\}$ is called a deviation functional if $\vartheta$ is continuous, nonnegative, homogeneous of degree zero, and satisfies $\vartheta(K)=0$ if and only if $K$ is an extremal body. Such functionals exist, and if $\vartheta$ is given, there exists 
(see [8]) a stability function for $\Sigma$ and $\vartheta$, that is, a continuous function $f:[0, \infty) \rightarrow$ $[0, \infty)$ with $f(0)=0$ and $f(\varepsilon)>0$ for $\varepsilon>0$ such that

$$
\vartheta(K) \geq \varepsilon \quad \text { implies } \quad \Phi(K) \geq(1+f(\varepsilon)) \tau \Sigma(K)^{1 / k},
$$

for $K \in \mathcal{K}^{d}$. We assume $f<1$, replacing, if necessary, $f$ by $\min \left\{f, \frac{1}{2}\right\}$.

We can now state our result on the typical cell $Z$ of the hyperplane tessellation induced by $X$. A formal definition of $Z$ is given in the next section. $\mathbb{P}(\cdot \mid \cdot)$ denotes a conditional probability.

Theorem. Suppose that a size functional $\Sigma$ with the listed properties, a deviation functional $\vartheta$, and a stability function $f$ for $\Sigma$ and $\vartheta$ are given. With a suitable constant $c_{0}>0$ (depending only on $\tau$ ), the following holds. If $\varepsilon>0$ and $0<a<b \leq \infty$, then

$$
\mathbb{P}(\vartheta(Z) \geq \varepsilon \mid \Sigma(Z) \in[a, b)) \leq c \exp \left\{-c_{0} f(\varepsilon) a^{1 / k} \lambda\right\},
$$

where $c$ is a constant depending only on $\Sigma, f$, and $\varepsilon$.

Since this result is of the same type as Theorem 1 in [8], it has similar consequences to the existence of limit shapes for large typical cells; this need not be carried out here. We remark, however, that the simplest conclusion from (6) is the relation

$$
\lim _{a \rightarrow \infty} \mathbb{P}(\vartheta(Z) \geq \varepsilon \mid \Sigma(Z) \geq a)=0,
$$

for any $\varepsilon>0$, showing that large typical cells have small deviation from extremal bodies.

The theorem holds for any size functional $\Sigma$ satisfying the listed assumptions. For the concrete cases particularly interesting us, the intrinsic volumes and the diameter, simple deviation functionals and stability functions can be given explicitly. For $\Sigma=V_{k}$, $k \in\{2, \ldots, d\}$, where the extremal bodies are the balls, the deviation from a ball is suitably measured by

$$
\vartheta(K)=\min \left\{\frac{R-r}{R+r}: r B^{d}+z \subset K \subset R B^{d}+z, r, R>0, z \in \mathbb{R}^{d}\right\} .
$$

A stability function is given by $f(\varepsilon)=\gamma \varepsilon^{(d+3) / 2}$, with a constant $\gamma$ depending only on the dimension (see [5]). If $\Sigma$ is the diameter $D$, we can choose

$$
\vartheta(K):=\min \left\{\alpha \geq 0: S \subset D(K)^{-1} K \subset S+\alpha B^{d}, S \text { a unit segment }\right\} .
$$

Then $f(\varepsilon)=\varepsilon^{2} / 2$ is a possible choice (see [8]).

For the proof of the theorem, we first establish a suitable explicit representation for the distribution of the typical cell $Z$. The rest of the proof then heavily depends on [8].

\section{The Typical Cell of a Poisson Hyperplane Tessellation}

Recall that $Y$ denotes the process of the $d$-dimensional cells of the tessellation induced by the stationary, isotropic Poisson hyperplane process $X$. Thus, $Y$ is a stationary particle 
process, and as such it has an intensity $\gamma^{(d)}$ and a grain or shape distribution $\mathbb{Q}_{0}$ (see, e.g., Section 4.2 and Chapter 6 of [24]). The intensity is given by

$$
\gamma^{(d)}=\kappa_{d}\left(\frac{\kappa_{d-1} \lambda}{\omega_{d}}\right)^{d}
$$

by (6.48) of [24]. The grain distribution depends on the choice of a center function, which serves for picking out a definite element from each translation class of convex bodies. The center function can be any measurable map $\boldsymbol{c}: \mathcal{P}_{0}^{d} \rightarrow \mathbb{R}^{d}$ satisfying $\boldsymbol{c}(P+\boldsymbol{x})=\boldsymbol{c}(P)+\boldsymbol{x}$ for all $P \in \mathcal{P}_{0}^{d}$ and $x \in \mathbb{R}^{d}$; here $\mathcal{P}_{0}^{d} \subset \mathcal{K}^{d}$ is the set of polytopes with interior points. Let $C^{d}:=\left[-\frac{1}{2}, \frac{1}{2}\right]^{d}$. The grain distribution of $Y$ with respect to the center function $\boldsymbol{c}$ is the (Borel) probability measure $\mathbb{Q}_{0}$ on $\mathcal{K}^{d}$ given by

$$
\gamma^{(d)} \mathbb{Q}_{0}(\mathcal{A})=\mathbb{E} \sum_{P \in Y} \mathbf{1}_{\mathcal{A}}\{P-\boldsymbol{c}(P)\} \mathbf{1}_{C^{d}}(\boldsymbol{c}(P))
$$

for Borel sets $\mathcal{A} \subset \mathcal{K}^{d}$. It also has an ergodic interpretation, namely

$$
\mathbb{Q}_{0}(\mathcal{A})=\lim _{r \rightarrow \infty} \frac{\sum_{P \in Y} \mathbf{1}_{\mathcal{A}}\{P-\boldsymbol{c}(P) \in \mathcal{A}\} \mathbf{1}_{r C^{d}}(\boldsymbol{c}(P))}{\sum_{P \in Y} \mathbf{1}_{r C^{d}}(\boldsymbol{c}(P))} \quad \text { almost surely. }
$$

We call $\mathbb{Q}_{0}$ the distribution of the typical cell of $Y$, and the typical cell $Z$ of $Y$ is any random polytope with distribution $\mathbb{Q}_{0}$. (The choice of the center function does not affect the shape of the typical cell: if $\boldsymbol{c}^{\prime}$ is another center function, then the corresponding distribution $\mathbb{Q}_{0}^{\prime}$ is the image measure of $\mathbb{Q}_{0}$ under the mapping $K \mapsto K-c^{\prime}(K)$.)

A representation of $Z$, using the center of the inball as a center function, was discussed by Miles [16], [18] and was extended and made more explicit by Calka [2]. We have, however, not succeeded in employing this for our intended result. More useful is a second representation, using the "lowest vertex" as a center function. We give a formula for the distribution of $Z$ with this center function, extending some related but less explicit results found in the literature [18], [1, Chapter 9], [3], [15], [12]. As did Calka [2], we use the Slivnyak-Mecke formula (following the terminology of Møller [22], see also [20] and [21]; a general version appears in [14]). Specialized to our Poisson hyperplane process, it says that, for $m \in \mathbb{N}$ and any nonnegative measurable function $f$ on $\mathbf{N} \times\left(\mathcal{H}^{d}\right)^{m}$ (where $\mathrm{N}$ is the measurable space of locally finite counting measures on $\mathcal{H}^{d}$ ),

$$
\begin{aligned}
\mathbb{E} \sum_{\left(H_{1}, \ldots, H_{m}\right) \in X_{\neq}^{m}} f\left(X ; H_{1}, \ldots, H_{m}\right) \\
\quad=\int_{\mathcal{H}^{d}} \cdots \int_{\mathcal{H}^{d}} \mathbb{E} f\left(X \cup\left\{H_{1}, \ldots, H_{m}\right\} ; H_{1}, \ldots, H_{m}\right) \Theta\left(\mathrm{d} H_{1}\right) \cdots \Theta\left(\mathrm{d} H_{m}\right) .
\end{aligned}
$$

Here, $X_{\neq}^{m}$ denotes the set of all $m$-tuples of pairwise different elements from $X$.

We fix a unit vector $\xi \in S^{d-1}$. For a polytope $P$, the lowest vertex $t(P)$ is the vertex of $P$ at which $\langle\xi, \cdot\rangle$ attains its minimum on $P$, if this vertex is unique. Suppose that this is the case, and that $P$ is simple. Then $\boldsymbol{t}(P)$ is contained in $d$ facet hyperplanes $H_{1}, \ldots, H_{d}$ of $P$; let $\boldsymbol{u}_{1}, \ldots, \boldsymbol{u}_{d}$ be their outer unit normal vectors. We have $\boldsymbol{\xi} \in$ int $\operatorname{pos}\left\{\boldsymbol{u}_{1}, \ldots, \boldsymbol{u}_{d}\right\}$, where pos denotes the positive hull and int is the interior. Conversely, if $v$ is a vertex of 
$P$ and the outer unit normal vectors $\boldsymbol{u}_{1}, \ldots, \boldsymbol{u}_{d}$ of the facets of $P$ containing $\boldsymbol{v}$ satisfy $\boldsymbol{\xi} \in$ int $\operatorname{pos}\left\{\boldsymbol{u}_{1}, \ldots, \boldsymbol{u}_{d}\right\}$, then $\boldsymbol{v}=\boldsymbol{t}(P)$.

Let $H_{1}, \ldots, H_{d}$ be hyperplanes with independent normal vectors. We denote by $\boldsymbol{s}\left(H_{1}, \ldots, H_{d}\right)$ their intersection point. Suppose that $\boldsymbol{\xi}$ is not in the linear hull of less than $d$ of the mentioned normal vectors. Then there is a unique choice of unit normal vectors $\boldsymbol{u}_{1}, \ldots, \boldsymbol{u}_{d}$ of the hyperplanes $H_{1}, \ldots, H_{d}$ such that $\boldsymbol{\xi} \in \operatorname{pos}\left\{\boldsymbol{u}_{1}, \ldots, \boldsymbol{u}_{d}\right\}$. We define a simplicial cone with apex $s\left(H_{1}, \ldots, H_{d}\right)$ by

$$
T\left(H_{1}, \ldots, H_{d}\right):=\bigcap_{i=1}^{d} H^{-}\left(\boldsymbol{u}_{i},\left\langle\boldsymbol{s}\left(H_{1}, \ldots, H_{d}\right), \boldsymbol{u}_{i}\right\rangle\right) .
$$

The stationary, isotropic Poisson hyperplane process $X$ has the property that almost surely any $d$ of its hyperplanes have linearly independent normal vectors and any $d-1$ hyperplanes have normal vectors which together with $\boldsymbol{\xi}$ are linearly independent (this can be proved by arguments similar to those applied in Theorem 4.1.6 of [24]). It follows that almost surely the cells of $Y$ (which are simple polytopes by the stationarity of $X$ ) have a unique lowest vertex. Let $P$ be a cell of $Y$. Its (almost surely existing) lowest vertex $\boldsymbol{t}(P)$ is the intersection of $d$ hyperplanes $H_{1}, \ldots, H_{d}$ of $X$, thus $\boldsymbol{t}(P)=\boldsymbol{s}\left(H_{1}, \ldots, H_{d}\right)$, and

$$
P=\bigcap_{H \in X \backslash\left\{H_{1}, \ldots, H_{d}\right\}} H^{-}\left(s\left(H_{1}, \ldots, H_{d}\right)\right) \cap T\left(H_{1}, \ldots, H_{d}\right) .
$$

Conversely, almost surely for every choice of different hyperplanes $H_{1}, \ldots, H_{d}$ from $X$, the right-hand side of (8) is a cell of $Y$, and $s\left(H_{1}, \ldots, H_{d}\right)$ is its lowest vertex.

Let $\mathbb{Q}_{0}$ be the distribution of the typical cell of $Y$ with respect to the lowest vertex as a center function. In the subsequent formulas, the arguments $H_{1}, \ldots, H_{d}$ of $s$ and $T$ are omitted, but have to be kept in mind. For Borel sets $\mathcal{A} \subset \mathcal{K}^{d}$, we obtain

$$
\begin{aligned}
\gamma^{(d)} \mathbb{Q}_{0}(\mathcal{A}) & =\mathbb{E} \sum_{P \in Y} \mathbf{1}_{\mathcal{A}}(P-\boldsymbol{t}(P)) \mathbf{1}_{C^{d}}(\boldsymbol{t}(P)) \\
& =\frac{1}{d !} \mathbb{E} \sum_{\left(H_{1}, \ldots, H_{d}\right) \in X_{\neq}^{d}} \mathbf{1}_{\mathcal{A}}\left(\bigcap_{H \in X \backslash\left\{H_{1}, \ldots, H_{d}\right\}}\left(H^{-}(\boldsymbol{s}) \cap T\right)-\boldsymbol{s}\right) \mathbf{1}_{C^{d}}(\boldsymbol{s}) \\
& =\frac{1}{d !} \int_{\mathcal{H}^{d}} \cdots \int_{\mathcal{H}^{d}} \mathbb{E} \mathbf{1}_{\mathcal{A}}\left(\bigcap_{H \in X}\left(H^{-}(\boldsymbol{s}) \cap T\right)-\boldsymbol{s}\right) \mathbf{1}_{C^{d}}(\boldsymbol{s}) \Theta\left(\mathrm{d} H_{1}\right) \cdots \Theta\left(\mathrm{d} H_{d}\right) \\
& =\frac{1}{d !} \int_{\mathcal{H}^{d}} \cdots \int_{\mathcal{H}^{d}} \mathbb{P}\left(Z_{0} \cap(T-\boldsymbol{s}) \in \mathcal{A}\right) \mathbf{1}_{C^{d}}(\boldsymbol{s}) \Theta\left(\mathrm{d} H_{1}\right) \cdots \Theta\left(\mathrm{d} H_{d}\right),
\end{aligned}
$$

where we have used the Slivnyak-Mecke formula, the stationarity of $X$, and the fact that $\bigcap_{H \in X} H^{-}(\boldsymbol{o})$ is the zero cell $Z_{0}$ of $Y$. We insert the representation of the intensity measure given by $\Theta=\lambda \mu$ and (1). Then we observe that

$$
T\left(H\left(\boldsymbol{u}_{1}, t_{1}\right), \ldots, H\left(\boldsymbol{u}_{d}, t_{d}\right)\right)-\boldsymbol{s}\left(H\left(\boldsymbol{u}_{1}, t_{1}\right), \ldots, H\left(\boldsymbol{u}_{d}, t_{d}\right)\right)=\bigcap_{j=1}^{d} H^{-}\left(\varepsilon_{j} \boldsymbol{u}_{j}, 0\right)
$$


if the factors $\varepsilon_{j} \in\{-1,1\}$ are chosen such that $\boldsymbol{\xi} \in \operatorname{pos}\left\{\varepsilon_{1} \boldsymbol{u}_{1}, \ldots, \varepsilon_{d} \boldsymbol{u}_{d}\right\}$. Such a choice is unique if $\boldsymbol{u}_{1}, \ldots, \boldsymbol{u}_{d}$ are linearly independent and $\boldsymbol{\xi}$ is not in the linear hull of less than $d$ of these vectors. We write the multiple integral as a sum of $2^{d}$ multiple integrals, each one extending over $\left(\boldsymbol{u}_{1}, \ldots, \boldsymbol{u}_{d}\right)$ with $\boldsymbol{\xi} \in \operatorname{pos}\left\{\varepsilon_{1} \boldsymbol{u}_{1}, \ldots, \varepsilon_{d} \boldsymbol{u}_{d}\right\}$ for a fixed $d$-tuple $\left(\varepsilon_{1}, \ldots, \varepsilon_{d}\right)$ (and over all $\left(t_{1}, \ldots, t_{d}\right)$ ). Noting that

$$
\boldsymbol{s}\left(H\left(\boldsymbol{u}_{1}, t_{1}\right), \ldots, H\left(\boldsymbol{u}_{d}, t_{d}\right)\right)=\boldsymbol{s}\left(H\left(\varepsilon_{1} \boldsymbol{u}_{1}, \varepsilon_{1} t_{1}\right), \ldots, H\left(\varepsilon_{d} \boldsymbol{u}_{d}, \varepsilon_{d} t_{d}\right)\right)
$$

and using the invariance of $\sigma$ and the one-dimensional Lebesgue measure under reflections in the origin, we obtain

$$
\begin{aligned}
\gamma^{(d)} \mathbb{Q}_{0}(\mathcal{A})= & \frac{(2 \lambda)^{d}}{d !} \int_{S^{d-1}} \cdots \int_{S^{d-1}} \int_{\mathbb{R}} \cdots \int_{\mathbb{R}} \mathbb{P}\left(Z_{0} \cap \bigcap_{j=1}^{d} H^{-}\left(\boldsymbol{u}_{j}, 0\right) \in \mathcal{A}\right) \\
& \times \mathbf{1}\left\{\boldsymbol{\xi} \in \operatorname{pos}\left\{\boldsymbol{u}_{1}, \ldots, \boldsymbol{u}_{d}\right\}\right\} \mathbf{1}_{C^{d}}(\boldsymbol{s}) \mathrm{d} t_{1} \cdots \mathrm{d} t_{d} \sigma\left(\mathrm{d} \boldsymbol{u}_{1}\right) \cdots \sigma\left(\mathrm{d} \boldsymbol{u}_{d}\right) .
\end{aligned}
$$

In the integrand, the symbol $s$ now denotes the intersection point of the hyperplanes $H\left(\boldsymbol{u}_{1}, t_{1}\right), \ldots, H\left(\boldsymbol{u}_{d}, t_{d}\right)$. For fixed linearly independent unit vectors $\boldsymbol{u}_{1}, \ldots, \boldsymbol{u}_{d}$, let $F\left(\boldsymbol{u}_{1}, \ldots, \boldsymbol{u}_{d}\right)$ denote this intersection point. This defines a bijective mapping $F$ from $\mathbb{R}^{d}$ to $\mathbb{R}^{d}$. Its inverse has Jacobian $\nabla_{d}\left(\boldsymbol{u}_{1}, \ldots, \boldsymbol{u}_{d}\right)$, the volume of the parallelepiped spanned by $\boldsymbol{u}_{1}, \ldots, \boldsymbol{u}_{d}$. Observing (7), we obtain the required result, which we formulate as a lemma.

Lemma 3.1. The distribution of the typical cell $Z$ of a stationary, isotropic Poisson hyperplane tessellation with respect to the lowest vertex in direction $\boldsymbol{\xi}$ as a center function is given by

$$
\begin{aligned}
\mathbb{P}(Z \in \mathcal{A})= & \frac{1}{d ! \kappa_{d}}\left(\frac{2 d \kappa_{d}}{\kappa_{d-1}}\right)^{d} \int_{S^{d-1}} \ldots \int_{S^{d-1}} \mathbb{P}\left(Z_{0} \cap \bigcap_{j=1}^{d} H^{-}\left(\boldsymbol{u}_{j}, 0\right) \in \mathcal{A}\right) \\
& \times \mathbf{1}\left\{\boldsymbol{\xi} \in \operatorname{pos}\left\{\boldsymbol{u}_{1}, \ldots, \boldsymbol{u}_{d}\right\}\right\} \nabla_{d}\left(\boldsymbol{u}_{1}, \ldots, \boldsymbol{u}_{d}\right) \sigma\left(\mathrm{d} \boldsymbol{u}_{1}\right) \cdots \sigma\left(\mathrm{d} \boldsymbol{u}_{d}\right),
\end{aligned}
$$

where $Z_{0}$ is the zero cell of the tessellation.

We remark that the isotropy assumption is not necessary here. The result extends to a stationary Poisson hyperplane tessellation, with a spherical direction distribution $\varphi$ not concentrated on a great subsphere. Since $\varphi$ can be assumed to be an even measure, the proof holds (with some care, including a suitable choice of $\boldsymbol{\xi}$ ), if $\sigma$ is replaced by $\varphi$ and the intensity $\gamma^{(d)}$ is evaluated according to (6.46) of [24].

\section{Proof of the Theorem}

We assume that $X, \Sigma, \vartheta$, and $f$ are given as explained in Section 2. The number $k$ is the degree of homogeneity of $\Sigma$. In the following, $c_{1}, c_{2}, \ldots$ denote constants depending only on $d$ and $\Sigma$, unless the dependence on additional quantities is explicitly indicated.

The first lemma for the proof of the theorem can be deduced from the corresponding lemma in [8]. 
Lemma 4.1. For each $\beta>0$, there are constants $h_{0}>0$ and $c>0$, depending only on $\Sigma$ and $\beta$, such that for $a^{1 / k} \lambda \geq 1$ and $0<h<h_{0}$,

$$
\mathbb{P}(\Sigma(Z) \in a(1,1+h)) \geq c h \exp \left\{-(1+\beta) \tau a^{1 / k} \lambda\right\} .
$$

Proof. By Lemma 1 of [8] (specialized to stationary, isotropic $X$ ), there exist constants $h_{0}>0, N \in \mathbb{N}(N \geq d$, without loss of generality), and $c>0$, depending only on $\Sigma$ and $\beta$, such that, for $a>0$ and $0<h<h_{0}$,

$$
\mathbb{P}\left(\Sigma\left(Z_{0}\right) \in a(1,1+h)\right) \geq \operatorname{ch}\left(a^{1 / k} \lambda\right)^{N} \exp \left\{-(1+\beta) \tau a^{1 / k} \lambda\right\} .
$$

The distributions of the typical cell $Z$ and the zero cell $Z_{0}$ are related in the following way (see, e.g., Theorem 6.1 .11 of [24]). For any translation invariant, nonnegative, measurable function $g$ on $\mathcal{K}^{d}$,

$$
\mathbb{E} g\left(Z_{0}\right)=\gamma^{(d)} \mathbb{E}\left[g(Z) V_{d}(Z)\right],
$$

where $\gamma^{(d)}=1 / \mathbb{E} V_{d}(Z)$ is given by (7). We apply this with

$$
g(K):=\mathbf{1}\{\Sigma(K) \in a(1,1+h)\} V_{d}(K)^{-1},
$$

observe that the function $\Sigma$ has the property that $V_{d}(K) \leq c_{1} \Sigma(K)^{d / k}$, assume $h<h_{0}$, and use (9) together with the assumption that $a^{1 / k} \lambda \geq 1$. This gives

$$
\begin{aligned}
\mathbb{P}(\Sigma(Z) \in a(1,1+h)) & =c_{2} \lambda^{-d} \mathbb{E}\left[\mathbf{1}\left\{\Sigma\left(Z_{0}\right) \in a(1,1+h)\right\} V_{d}\left(Z_{0}\right)^{-1}\right] \\
& \geq c_{2} \lambda^{-d} \mathbb{E}\left[\mathbf{1}\left\{\Sigma\left(Z_{0}\right) \in a(1,1+h)\right\} c_{1}^{-1}(a(1+h))^{-d / k}\right] \\
& \geq c_{3}(\beta)\left(a^{1 / k} \lambda\right)^{-d} \mathbb{P}\left(\Sigma\left(Z_{0}\right) \in a(1,1+h)\right) \\
& \geq c_{4}(\beta) h \exp \left\{-(1+\beta) \tau a^{1 / k} \lambda\right\},
\end{aligned}
$$

completing the proof of the lemma.

Further proof of the theorem now connects the distribution of the typical cell $Z$ with that of the zero cell $Z_{0}$ by means of Lemma 3.1. In particular, this lemma gives, for $\varepsilon \geq 0$,

$$
\begin{aligned}
\mathbb{P}(\Sigma(Z) \in & a(1,1+h), \vartheta(Z) \geq \varepsilon) \\
= & \frac{1}{d ! \kappa_{d}}\left(\frac{2 d \kappa_{d}}{\kappa_{d-1}}\right)^{d} \\
& \times \int_{S^{d-1}} \cdots \int_{S^{d-1}} \mathbb{P}\left(\Sigma\left(Z_{0} \cap T\right) \in a(1,1+h), \vartheta\left(Z_{0} \cap T\right) \geq \varepsilon\right) \\
& \times \mathbf{1}\left\{\boldsymbol{\xi} \in \operatorname{pos}\left\{\boldsymbol{u}_{1}, \ldots, \boldsymbol{u}_{d}\right\}\right\} \nabla_{d}\left(\boldsymbol{u}_{1}, \ldots, \boldsymbol{u}_{d}\right) \sigma\left(\mathrm{d} \boldsymbol{u}_{1}\right) \cdots \sigma\left(\mathrm{d} \boldsymbol{u}_{d}\right),
\end{aligned}
$$

where now

$$
T=T\left(\boldsymbol{u}_{1}, \ldots, \boldsymbol{u}_{d}\right):=\bigcap_{j=1}^{d} H^{-}\left(\boldsymbol{u}_{j}, 0\right) .
$$


For given hyperplanes $H_{1}, \ldots, H_{n} \in \mathcal{H}^{d}$, we write

$$
P_{T}\left(H_{(n)}\right):=H_{1}^{-} \cap \cdots \cap H_{n}^{-} \cap T .
$$

The idea is now to apply the methods of [8] to $Z_{0} \cap T$ instead of $Z_{0}$. This requires some adaptations, which we carry out with just the amount of detail as seems necessary for an understanding. Some definitions need to be repeated.

We assume for a while that linearly independent unit vectors $\boldsymbol{u}_{1}, \ldots, \boldsymbol{u}_{d} \in S^{d-1}$ with $\boldsymbol{\xi} \in \operatorname{pos}\left\{\boldsymbol{u}_{1}, \ldots, \boldsymbol{u}_{d}\right\}$ are fixed, the simplicial cone $T$ is defined by (11), and we put $Z_{T}:=Z_{0} \cap T$ and $E_{i}:=H\left(\boldsymbol{u}_{i}, 0\right)$ for $i=1, \ldots, d$.

For $K \in \mathcal{K}^{d}$ with $\Sigma(K)>0$, the relative diameter is defined by $\Delta(K):=D(K) /$ $c_{5} \Sigma(K)^{1 / k}$, where $c_{5}$ is chosen such that $\Delta(K) \geq 1$ for all $K$. For $a>0, \varepsilon \geq 0, h>0$, and $m \in \mathbb{N}$ we define

$$
\mathcal{K}_{a, \varepsilon, h}(m):=\left\{K \in \mathcal{K}^{d}: \Sigma(K) \in a(1,1+h), \vartheta(K) \geq \varepsilon, \Delta(K) \in[m, m+1)\right\} .
$$

The following is Lemma 2 from [8].

Lemma 4.2. Let $m \in \mathbb{N}$. Then $K \in \mathcal{K}_{a, 0,1}(m)$ and $\boldsymbol{o} \in K$ implies $K \subset c_{6} m a^{1 / k} B^{d}=$ : $C$. There exists a measurable map that associates with every polytope $P \in \mathcal{K}_{a, 0,1}(m)$ with $\boldsymbol{o} \in P$ a vertex $\boldsymbol{v}(P)$ of $P$ with $\|\boldsymbol{v}(P)\| \geq c_{7} m a^{1 / k}$.

Now let

$$
q_{a, \varepsilon, h}(m):=\mathbb{P}\left(Z_{T} \in \mathcal{K}_{a, \varepsilon, h}(m)\right)
$$

then

$$
\sum_{m=1}^{\infty} q_{a, \varepsilon, h}(m)=\mathbb{P}\left(\Sigma\left(Z_{T}\right) \in a(1,1+h), \vartheta\left(Z_{T}\right) \geq \varepsilon\right) .
$$

For given $m \in \mathbb{N}$ and $a>0$, let $C$ be the ball according to Lemma 4.2. We have

$$
q_{a, \varepsilon, 1}(m)=\sum_{N=1}^{\infty} \mathbb{P}\left(X\left(\mathcal{H}_{C}\right)=N\right) \mathbb{P}\left(Z_{T} \in \mathcal{K}_{a, \varepsilon, 1}(m) \mid X\left(\mathcal{H}_{C}\right)=N\right) .
$$

Here,

$$
\begin{aligned}
p_{N} & :=\mathbb{P}\left(Z_{T} \in \mathcal{K}_{a, \varepsilon, 1}(m) \mid X\left(\mathcal{H}_{C}\right)=N\right) \\
& =\Phi(C)^{-N} \int_{\mathcal{H}_{C}^{N}} \mathbf{1}\left\{P_{T}\left(H_{(N)}\right) \in \mathcal{K}_{a, \varepsilon, 1}(m)\right\} \mu^{N}\left(\mathrm{~d}\left(H_{1}, \ldots, H_{N}\right)\right)
\end{aligned}
$$

with

$$
\mathcal{H}_{C}^{N}:=\mathcal{H}_{C} \times \cdots \times \mathcal{H}_{C} \quad \text { and } \quad \mu^{N}:=\mu \otimes \cdots \otimes \mu \quad(N \text { factors }) .
$$

Relation (14) holds since $X$ is a Poisson process with intensity measure $\lambda \mu$, and $\mu\left(\mathcal{H}_{C}\right)=\Phi(C)$.

Now we adapt Lemma 3 from [8] to the random polytope $Z_{T}$ instead of $Z_{0}$. 
Lemma 4.3. For $a>0$ and $m \in \mathbb{N}$,

$$
q_{a, 0,1}(m) \leq c_{11} \exp \left\{-c_{9} m a^{1 / k} \lambda\right\}
$$

Proof. Let $a>0$, let $m \in \mathbb{N}$, and let $C$ be the ball defined in Lemma 4.2. Let $H_{1}, \ldots, H_{N} \in \mathcal{H}_{C}$ be hyperplanes such that $P:=P_{T}\left(H_{(N)}\right) \in \mathcal{K}_{a, 0,1}(m)$. Let $\boldsymbol{v}(P)$ be the vertex defined in Lemma 4.2. This vertex is the intersection of $d$ facets of $P$, and it is different from $\boldsymbol{o}$. Hence, there is a number $e \in\{0, \ldots, d-1\}$, and there are index sets $I \subset\{1, \ldots, d\}$ with $e$ elements and $J \subset\{1, \ldots, N\}$ with $d-e$ elements such that

$$
\{v(P)\}=\bigcap_{i \in I} E_{i} \cap \bigcap_{j \in J} H_{j} .
$$

We denote the segment $[\boldsymbol{o}, \boldsymbol{v}(P)]$ by $S=S\left(E_{I}, H_{J}\right)$. It satisfies

$$
H_{r} \cap \operatorname{relint} S=\emptyset \quad \text { for } \quad r \in\{1, \ldots, N\} \backslash J,
$$

where relint denotes the relative interior. Since $S \subset C$ and $\Phi(S)$ is a constant multiple of the length $|S|$ of $S$, we have

$$
\int_{\mathcal{H}_{C}} \mathbf{1}\{H \cap S=\emptyset\} \mu(\mathrm{d} H)=\Phi(C)-\Phi(S)=\Phi(C)-c_{8}|S| \leq \Phi(C)-2 c_{9} m a^{1 / k} .
$$

Defining

$$
\begin{aligned}
p_{N}(e):= & \Phi(C)^{-N} \int_{\mathcal{H}_{C}^{N}} \mathbf{1}\left\{P_{T}\left(H_{(N)}\right) \in \mathcal{K}_{a, 0,1}(m)\right\} \\
& \times \mathbf{1}\left\{\operatorname{card}\left\{i: \boldsymbol{v}\left(P_{T}\left(H_{(N)}\right)\right) \in E_{i}\right\}=e\right\} \mu^{N}\left(\mathrm{~d}\left(H_{1}, \ldots, H_{N}\right)\right),
\end{aligned}
$$

we obtain

$$
\begin{aligned}
p_{N}(e) \leq & \left(\begin{array}{c}
d \\
e
\end{array}\right)\left(\begin{array}{c}
N \\
d-e
\end{array}\right) \Phi(C)^{-N} \int_{\mathcal{H}_{C}^{d-e}} \int_{\mathcal{H}_{C}^{N-d+e}} \mathbf{1}\left\{\left|S\left(E_{\{1, \ldots, e\}}, H_{\{1, \ldots, d-e\}}\right)\right| \geq c_{7} m a^{1 / k}\right\} \\
& \times \mathbf{1}\left\{S\left(E_{\{1, \ldots, e\}}, H_{\{1, \ldots, d-e\}}\right) \cap H_{r}=\emptyset \text { for } r=d-e+1, \ldots, N\right\} \\
& \times \mu^{N-d+e}\left(\mathrm{~d}\left(H_{d-e+1}, \ldots, H_{N}\right)\right) \mu^{d-e}\left(\mathrm{~d}\left(H_{1}, \ldots, H_{d-e}\right)\right) \\
\leq & \left(\begin{array}{c}
d \\
e
\end{array}\right)\left(\begin{array}{c}
N \\
d-e
\end{array}\right) \Phi(C)^{-N} \\
& \times \int_{\mathcal{H}_{C}^{d-e}}\left[\Phi(C)-2 c_{9} m a^{1 / k}\right]^{N-d+e} \mu^{d-e}\left(\mathrm{~d}\left(H_{1}, \ldots, H_{d-e}\right)\right) \\
= & \left(\begin{array}{c}
d \\
e
\end{array}\right)\left(\begin{array}{c}
N \\
d-e
\end{array}\right) \Phi(C)^{d-e-N}\left[\Phi(C)-2 c_{9} m a^{1 / k}\right]^{N-d+e} .
\end{aligned}
$$


Summing over $e$ and $N$, we get

$$
\begin{aligned}
q_{a, 0,1}(m) \leq & \sum_{e=0}^{d-1} \sum_{N=d-e}^{\infty} \frac{[\Phi(C) \lambda]^{N}}{N !} \mathrm{e}^{-\Phi(C) \lambda} \\
& \times\left(\begin{array}{c}
d \\
e
\end{array}\right)\left(\begin{array}{c}
N \\
d-e
\end{array}\right) \Phi(C)^{d-e-N}\left[\Phi(C)-2 c_{9} m a^{1 / k}\right]^{N-d+e} \\
= & \sum_{e=0}^{d-1} \frac{1}{(d-e) !}\left(\begin{array}{l}
d \\
e
\end{array}\right)[\Phi(C) \lambda]^{d-e} \mathrm{e}^{-\Phi(C) \lambda} \\
& \times \sum_{N=d-e}^{\infty} \frac{1}{(N-d+e) !}\left[\Phi(C) \lambda-2 c_{9} m a^{1 / k} \lambda\right]^{N-d+e} \\
= & \sum_{e=0}^{d-1} \frac{1}{(d-e) !}\left(\begin{array}{l}
d \\
e
\end{array}\right)[\Phi(C) \lambda]^{d-e} \exp \left\{-2 c_{9} m a^{1 / k} \lambda\right\} \\
\leq & \sum_{e=0}^{d-1} c_{10}\left(m a^{1 / k} \lambda\right)^{d-e} \exp \left\{-2 c_{9} m a^{1 / k} \lambda\right\} \\
\leq & c_{11} \exp \left\{-c_{9} m a^{1 / k} \lambda\right\},
\end{aligned}
$$

as stated.

We quote Lemma 4 from [8]. Here ext $P$ and $f_{0}(P)$ denote, respectively, the set and the number of vertices of the polytope $P$. Then we adapt Lemma 5 from [8].

Lemma 4.4. Let $\alpha>0$ be given. There is a number $v \in \mathbb{N}$ depending only on $d$ and $\alpha$ such that the following is true. For $P \in \mathcal{P}^{d}$ there exists a polytope $Q=Q(P) \in \mathcal{P}^{d}$ satisfying ext $Q \subset$ ext $P, f_{0}(Q) \leq v$, and $\Phi(Q) \geq(1-\alpha) \Phi(P)$. Moreover, there exists a measurable selection $P \mapsto Q(P)$.

Lemma 4.5. For $a>0, m \in \mathbb{N}$, and $\varepsilon>0$,

$$
q_{a, \varepsilon, 1}(m) \leq c_{14}(f, \varepsilon) m^{d v} \exp \left\{-(1+f(\varepsilon) / 3) \tau a^{1 / k} \lambda\right\},
$$

where $v$ depends only on $d$ and $\varepsilon$.

Proof. Let $B$ be an extremal body for the functional $\Sigma$, and let $B_{a}$ be the dilate of $B$ with $\Sigma\left(B_{a}\right)=a$. Let $m \in \mathbb{N}$ be given, and let $C$ be the ball from Lemma 4.2. We use (13) and (14). Suppose that $H_{1}, \ldots, H_{N} \in \mathcal{H}_{C}$ are such that $P_{T}\left(H_{(N)}\right) \in \mathcal{K}_{a, \varepsilon, 1}(m)$. By (5) and since $\Phi\left(B_{a}\right)=\tau \Sigma\left(B_{a}\right)^{1 / k}=\tau a^{1 / k}$, we get

$$
\begin{aligned}
\Phi\left(P_{T}\left(H_{(N)}\right)\right) & \geq(1+f(\varepsilon)) \tau \Sigma\left(P_{T}\left(H_{(N)}\right)\right)^{1 / k} \geq(1+f(\varepsilon)) \tau a^{1 / k} \\
& =(1+f(\varepsilon)) \Phi\left(B_{a}\right) .
\end{aligned}
$$

Let $\alpha:=f(\varepsilon) /(2+f(\varepsilon))$, then $(1-\alpha)(1+f(\varepsilon))=1+\alpha$. 
By Lemma 4.4, there are $v=v(d, \varepsilon)$ vertices of $P_{T}\left(H_{(N)}\right)$ such that the convex hull $Q\left(P_{T}\left(H_{(N)}\right)\right)=: Q\left(H_{(N)}\right)=: Q$ of these vertices satisfies

$$
\Phi(Q) \geq(1-\alpha) \Phi\left(P_{T}\left(H_{(N)}\right)\right) .
$$

Together with (15), this implies

$$
\Phi(Q) \geq(1+\alpha) \Phi\left(B_{a}\right) .
$$

For each $N$-tuple $\left(H_{1}, \ldots, H_{N}\right)$ such that $P_{T}\left(H_{(N)}\right) \in \mathcal{K}_{a, \varepsilon, 1}(m)$, we can choose $Q=$ $Q\left(H_{(N)}\right)$ in such a way that $Q\left(H_{(N)}\right)$ becomes a measurable function of $\left(H_{1}, \ldots, H_{N}\right)$. We can assume (excluding a set of $N$-tuples $\left(H_{1}, \ldots, H_{N}\right)$ of measure zero) that each of the vertices of $Q$ lies in precisely $d$ of the hyperplanes $E_{1}, \ldots, E_{d}, H_{1}, \ldots, H_{N}$, and the remaining hyperplanes of $H_{1}, \ldots, H_{N}$ are disjoint from $Q$. Hence, at most $d v$ of the hyperplanes $H_{1}, \ldots, H_{N}$ meet $Q$; let $j \in\{1, \ldots, d \nu\}$ denote their precise number. Suppose that $H_{1}, \ldots, H_{j}$ are the hyperplanes meeting $Q$. Then there is a sequence of pairs $\left(\left(I_{1}, J_{1}\right), \ldots,\left(I_{f_{0}}(Q), J_{f_{0}}(Q)\right)\right)$, where $I_{r}$ is a subset of $\{1, \ldots, d\}$ with $e_{r}$ elements and $J_{r}$ is a subset of $\{1, \ldots, j\}$ with $d-e_{r}$ elements, such that the intersections

$$
\bigcap_{i \in I_{r}} E_{i} \cap \bigcap_{j \in J_{r}} H_{j}, \quad r=1, \ldots, f_{0}(Q) \leq v,
$$

yield the vertices of $Q$. Below, the sum $\sum_{\left.\left(\left(I_{1}, J_{1}\right), \ldots, I_{v}, J_{v}\right)\right)}$ extends over all sequences $\left(\left(I_{1}, J_{1}\right), \ldots,\left(I_{v}, J_{v}\right)\right)$ of (not necessarily distinct) pairs, where $I_{r}$ is a subset of $\{1, \ldots, d\}, J_{r}$ is a subset of $\{1, \ldots, j\}$, and card $I_{r}+\operatorname{card} J_{r}=d$. The total number of such sequences can be estimated by a constant $c(d, v)$. We recall the fact that for any convex body $K \subset C$ we have $\int_{\mathcal{H}_{C}} \mathbf{1}\{H \cap K=\emptyset\} \mu(\mathrm{d} H)=\Phi(C)-\Phi(K)$. We get

$$
\begin{aligned}
& \mathbb{P}\left(Z_{T} \in \mathcal{K}_{a, \varepsilon, 1}(m) \mid X\left(\mathcal{H}_{C}\right)=N\right) \Phi(C)^{N} \\
& \leq \sum_{j=1}^{d v}\left(\begin{array}{c}
N \\
j
\end{array}\right) \\
& \times \int_{\mathcal{H}_{C}^{N}} \mathbf{1}\left\{P_{T}\left(H_{(N)}\right) \in \mathcal{K}_{a, \varepsilon, 1}(m)\right\} \mathbf{1}\left\{H_{s} \cap Q\left(H_{(N)}\right) \neq \emptyset \text { for } s=1, \ldots, j\right\} \\
& \times \mathbf{1}\left\{H_{s} \cap Q\left(H_{(N)}\right)=\emptyset \text { for } s=j+1, \ldots, N\right\} \mu^{N}\left(\mathrm{~d}\left(H_{1}, \ldots, H_{N}\right)\right) \\
& \leq \sum_{j=1}^{d v}\left(\begin{array}{c}
N \\
j
\end{array}\right) \sum_{\left(\left(I_{1}, J_{1}\right), \ldots,\left(I_{v}, J_{v}\right)\right)} \int_{\mathcal{H}_{C}^{j}} \int_{\mathcal{H}_{C}^{N-j}} \mathbf{1}\left\{\Phi\left(\operatorname{conv} \bigcup_{r=1}^{v}\left[\bigcap_{i \in I_{r}} E_{i} \cap \bigcap_{j \in J_{r}} H_{j}\right]\right)\right. \\
& \left.\geq(1+\alpha) \Phi\left(B_{a}\right)\right\} \\
& \times \mathbf{1}\left\{H_{s} \cap \operatorname{conv} \bigcup_{r=1}^{v}\left[\bigcap_{i \in I_{r}} E_{i} \cap \bigcap_{j \in J_{r}} H_{j}\right]=\emptyset \text { for } s=j+1, \ldots, N\right\} \\
& \times \mu^{N-j}\left(\mathrm{~d}\left(H_{j+1}, \ldots, H_{N}\right)\right) \mu^{j}\left(\mathrm{~d}\left(H_{1}, \ldots, H_{j}\right)\right) \\
& \leq \sum_{j=1}^{d v}\left(\begin{array}{c}
N \\
j
\end{array}\right) c(d, v)\left[\Phi(C)-(1+\alpha) \Phi\left(B_{a}\right)\right]^{N-j} \Phi(C)^{j} .
\end{aligned}
$$


Summation over $N$ gives

$$
\begin{aligned}
q_{a, \varepsilon, 1}(m) \leq & \sum_{N=1}^{\infty} \frac{[\Phi(C) \lambda]^{N}}{N !} \mathrm{e}^{-\Phi(C) \lambda} \sum_{j=1}^{d v}\left(\begin{array}{c}
N \\
j
\end{array}\right) c(d, v) \frac{\left[\Phi(C)-(1+\alpha) \Phi\left(B_{a}\right)\right]^{N-j}}{\Phi(C)^{N-j}} \\
= & \sum_{j=1}^{d v} c(d, v) \frac{[\Phi(C) \lambda]^{j}}{j !} \mathrm{e}^{-\Phi(C) \lambda} \\
& \times \sum_{N=j}^{\infty} \frac{1}{(N-j) !}\left[\Phi(C) \lambda-(1+\alpha) \Phi\left(B_{a}\right) \lambda\right]^{N-j} \\
= & \sum_{j=1}^{d v} c(d, v) \frac{[\Phi(C) \lambda]^{j}}{j !} \exp \left\{-(1+\alpha) \Phi\left(B_{a}\right) \lambda\right\} .
\end{aligned}
$$

Here $\Phi\left(B_{a}\right)=\tau a^{1 / k}$, and by Lemma $4.2, \Phi(C)=\Phi\left(c_{6} m a^{1 / k} B^{d}\right)=2 c_{6} m a^{1 / k}$. Thus we get

$$
\begin{aligned}
q_{a, \varepsilon, 1}(m) & \leq c_{12}(\varepsilon)\left[\left(a^{1 / k} \lambda\right)^{d v}+1\right] m^{d v} \exp \left\{-(1+\alpha) \tau a^{1 / k} \lambda\right\} \\
& \leq c_{13}(\varepsilon) m^{d v} \exp \left\{-(1+f(\varepsilon) / 3) \tau a^{1 / k} \lambda\right\}
\end{aligned}
$$

since $f(\varepsilon)<1$. This completes the proof of Lemma 4.5.

For the following parts of the proof, it suffices to describe the changes that the proof in [8] has to undergo. Lemma 6 of [8] remains unchanged; Lemma 7 is altered as follows (we number the counterpart to Lemma $x$ in [8] as Lemma 4. $x$ here). If $P$ is a polytope which arises by intersecting some polytope with the cone $T$, we denote by $f_{d-1}^{\prime}(P)$ the number of facets of $P$ not lying in one of the hyperplanes $E_{1}, \ldots, E_{d}$.

Lemma 4.7. For $n \in \mathbb{N}, n \geq 1$, and a Borel set $\mathcal{B} \subset \mathcal{K}^{d}$, let

$$
R(\mathcal{B}, n):=\left\{\left(H_{1}, \ldots, H_{n}\right) \in\left(\mathcal{H}^{d}\right)^{n}: P_{T}\left(H_{(n)}\right) \in \mathcal{B}, f_{d-1}^{\prime}\left(P_{T}\left(H_{(n)}\right)\right)=n\right\} .
$$

Then

$$
\mathbb{P}\left(Z_{T} \in \mathcal{B}, f_{d-1}^{\prime}\left(Z_{T}\right)=n\right)=\frac{\lambda^{n}}{n !} \int_{R(\mathcal{B}, n)} \exp \left\{-\Phi\left(P_{T}\left(H_{(n)}\right)\right) \lambda\right\} \mu^{n}\left(\mathrm{~d}\left(H_{1}, \ldots, H_{n}\right)\right) .
$$

The proof from [8] goes through with the indicated changes.

Lemma 4.8. For $m \in \mathbb{N}, h \in\left(0, \frac{1}{2}\right), \varepsilon \geq 0$, and $a^{1 / k} \lambda \geq 1$,

$$
q_{a, \varepsilon, h}(m) \leq c_{15} h a^{1 / k} \lambda m q_{a, \varepsilon, 1}(m) .
$$

The corresponding proof in [8] goes through if the definition of the set $R_{\varepsilon}(m, n)$ is changed to

$$
\begin{aligned}
R_{\varepsilon}(m, n):= & \left\{\left(H_{1}, \ldots, H_{n}\right) \in\left(\mathcal{H}^{d}\right)^{n}: \vartheta\left(P_{T}\left(H_{(n)}\right)\right) \geq \varepsilon, \Delta\left(P_{T}\left(H_{(n)}\right)\right) \in[m, m+1),\right. \\
& \left.f_{d-1}^{\prime}\left(P_{T}\left(H_{(n)}\right)\right)=n\right\},
\end{aligned}
$$


and each of the sets

$$
H^{-}\left(\boldsymbol{u}_{1}, t_{1}\right) \cap \cdots \cap H^{-}\left(\boldsymbol{u}_{n}, t_{n}\right), \quad H_{1}^{-} \cap \cdots \cap H_{n-1}^{-} \cap H^{-}(\boldsymbol{u}, 1),
$$

wherever it occurs, is replaced by its intersection with $T$. Since $T$ is a cone, the modified set $R_{\varepsilon}(m, n)$ is also invariant under dilatations applied to $H_{1}, \ldots, H_{n}$, which is crucial for the proof.

The following lemma finally concerns the typical cell $Z$.

Lemma 4.9. Let $\varepsilon>0, h \in\left(0, \frac{1}{2}\right)$, and $a^{1 / k} \lambda \geq 1$. Then

$$
\mathbb{P}(\Sigma(Z) \in a(1,1+h), \vartheta(Z) \geq \varepsilon) \leq c_{17}(f, \varepsilon) h \exp \left\{-(1+f(\varepsilon) / 4) \tau a^{1 / k} \lambda\right\} .
$$

Proof. First, we consider the random polytope $Z_{T}=Z_{0} \cap T$, where $T$ is the simplicial cone appearing above. With the constant $c_{9}$ from Lemma 4.3, we can choose $m_{0} \in \mathbb{N}$ such that

$$
c_{9} m \geq 2(1+f(\varepsilon) / 3) \tau \quad \text { for } m>m_{0}
$$

(recall that $f(\varepsilon)<1)$. By (12) and Lemma 4.8, we have

$$
\begin{aligned}
\mathbb{P}\left(\Sigma\left(Z_{T}\right) \in\right. & \left.a(1,1+h), \vartheta\left(Z_{T}\right) \geq \varepsilon\right) \\
& =\sum_{m \in \mathbb{N}} q_{a, \varepsilon, h}(m) \\
& \leq c_{15} h a^{1 / k} \lambda\left(\sum_{m=1}^{m_{0}} m q_{a, \varepsilon, 1}(m)+\sum_{m>m_{0}} m q_{a, \varepsilon, 1}(m)\right) .
\end{aligned}
$$

For the estimation of $q_{a, \varepsilon, 1}(m)$ we use Lemma 4.5 for $m \leq m_{0}$ and Lemma 4.3 for $m>m_{0}$, observing that $q_{a, \varepsilon, 1}(m) \leq q_{a, 0,1}(m)$. Then we can continue in the same way as in the proof of Proposition 7.1 in [4], where (24) of [4] is replaced by (16). In this way, we obtain the estimate

$\mathbb{P}\left(\Sigma\left(Z_{0} \cap T\right) \in a(1,1+h), \vartheta\left(Z_{0} \cap T\right) \geq \varepsilon\right) \leq c_{16}(f, \varepsilon) h \exp \left\{-(1+f(\varepsilon) / 4) \tau a^{1 / k} \lambda\right\}$.

This result holds for all cones $T$ appearing in the integral (10), up to a set of $d$-tuples $\left(\boldsymbol{u}_{1}, \ldots, \boldsymbol{u}_{d}\right)$ of measure zero. Therefore, formula (10) and integration yields the assertion of the lemma, with an appropriate constant.

The situation is now as in [8]: the proof of the theorem is completed in the same way as the proof of Theorem 1 in [4]. The latter used only Lemma 3.2 and Proposition 7.1 of [4], and our present Lemmas 4.1 and 4.9 have the same structure as those results. Since we have assumed $a^{1 / k} \lambda \geq 1$ in Lemmas 4.1 and 4.9, (6) is first obtained under this assumption. If we choose the constant $c$ appropriately, then (6) holds generally.

\section{References}

1. R.V. Ambartzumian, Factorization Calculus and Geometric Probability, Cambridge University Press, Cambridge, 1990.

2. P. Calka, Mosaïques poissoniennes de l'espace euclidien. Une extension d'un résultat de R.E. Miles, $C . R$. Acad. Sci. Paris Sér. I Math. 332 (2001), 557-562. 
3. W. Favis and V. Weiß, Mean values of weighted cells of stationary Poisson hyperplane tessellations of $\mathbb{R}^{d}$, Math. Nachr. 193 (1998), 37-48.

4. D. Hug, M. Reitzner, and R. Schneider, The limit shape of the zero cell in a stationary Poisson hyperplane tessellation, Ann. Probab. 32 (2004), 1140-1167.

5. D. Hug, M. Reitzner, and R. Schneider, Large Poisson-Voronoi cells and Crofton cells, Adv. in Appl. Prob. (SGSA) 36 (2004), 667-690.

6. D. Hug and R. Schneider, Large cells in Poisson-Delaunay tessellations, Discrete Comput. Geom. 31 (2004), 503-514.

7. D. Hug and R. Schneider, Large typical cells in Poisson-Delaunay mosaics, Rev. Roumaine Math. Pures Appl. 50 (2005), 657-670.

8. D. Hug and R. Schneider, Asymptotic shapes of large cells in random tessellations, Geom. Funct. Anal. 17 (2007), 156-191.

9. I.N. Kovalenko, A proof of a conjecture of David Kendall on the shape of random polygons of large area (Russian), Kibernet. Sistem. Anal. 1997, no. 4, 3-10, 187, Engl. transl. Cybernet. Systems Anal. 33 (1997), 461-467.

10. I.N. Kovalenko, An extension of a conjecture of D.G. Kendall concerning shapes of random polygons to Poisson Voronoï cells, in Engel, P., Syta, H. (eds.), Voronoi’s Impact on Modern Science, Book I. Transl. from the Ukrainian. Kyiv: Institute of Mathematics. Proc. Inst. Math. Natl. Acad. Sci. Ukr., Math. Appl. 21(1), 266-274 (1998).

11. I.N. Kovalenko, A simplified proof of a conjecture of D.G. Kendall concerning shapes of random polygons, J. Appl. Math. Stochastic Anal. 12 (1999), 301-310.

12. R. Maier, J. Mayer, and V. Schmidt, Distributional properties of the typical cell of stationary iterated tessellations, Math. Methods Oper. Res. 59 (2004), 287-302.

13. G. Matheron, Random Sets and Integral Geometry, Wiley, New York, 1975.

14. J. Mecke, Stationäre zufällige Maße auf lokalkompakten Abelschen Gruppen, Z. Wahrsch. Verw. Gebiete 9 (1967), 36-58

15. J. Mecke, On the relationship between the 0 -cell and the typical cell of a stationary random tessellation, Pattern Recognition 32 (1999), 1645-1648.

16. R.E. Miles, Random polytopes: the generalisation to $n$ dimensions of the intervals of a Poisson process, Thesis, Cambridge University, 1961.

17. R.E. Miles, A synopsis of "Poisson flats in Euclidean spaces", Izv. Akad. Nauk Armyan. SSR Ser. Mat. 5 (1970), 263-285; reprinted in Stochastic Geometry (E.F. Harding, D.G. Kendall, eds.) Wiley, New York, 1974, pp. 202-227.

18. R.E. Miles, The various aggregates of random polygons determined by random lines in a plane, Adv. Math. 10 (1973), 256-290.

19. R.E. Miles, A heuristic proof of a long-standing conjecture of D.G. Kendall concerning the shapes of certain large random polygons, Adv. in Appl. Prob. 27 (1995), 397-417.

20. J. Møller, Random tessellations in $\mathbb{R}^{d}$, Adv. in Appl. Prob. 21 (1989), 3-73.

21. J. Møller, Lectures on Random Voronoi Tessellations, Lecture Notes in Statistics, vol. 87, Springer, New York, 1994.

22. J. Møller, A review on probabilistic models and results for Voronoi tessellations, in Engel, P., Syta, H. (eds.), Voronoi's Impact on Modern Science, Book I. Transl. from the Ukrainian. Kyiv: Institute of Mathematics. Proc. Inst. Math. Natl. Acad. Sci. Ukr., Math. Appl. 21(1), 254-265 (1998).

23. R. Schneider, Convex Bodies—the Brunn-Minkowski Theory, Cambridge University Press, Cambridge, 1993.

24. R. Schneider and W. Weil, Stochastische Geometrie, Teubner, Stuttgart, 2000.

25. D. Stoyan, W.S. Kendall, and J. Mecke, Stochastic Geometry and Its Applications, 2nd edn., Wiley, Chichester, 1995.

Received February 27, 2006, and in revised form April 10, 2007. Online publication July 13, 2007. 